\title{
SPATIALLY RESTRICTED INTEGRALS IN GRADIOMETRIC BOUNDARY VALUE PROBLEMS
}

\author{
M. Eshagh \\ Division of Geodesy, Royal Institute of Technology (KTH), Stockholm, Sweden \\ Fax:+46 8 7907343; Tel: +4687907369 \\ Email:eshagh@kth se
}

\begin{abstract}
The spherical Slepian functions can be used to localize the solutions of the gradiometric boundary value problems on a sphere. These functions involve spatially restricted integral products of scalar, vector and tensor spherical harmonics. This paper formulates these integrals in terms of combinations of the Gaunt coefficients and integrals of associated Legendre functions. The presented formulas for these integrals are useful in recovering the Earth's gravity field locally from the satellite gravity gradiometry data.
\end{abstract}

Keywords Clebsch-Gordan's coefficients, Gaunt's coefficients, harmonic analysis, associated Legendre's function, satellite gravity gradiometry, Slepians function

\section{INTRODUCTION}

A boundary value problem (BVP) is a way of formulating the mathematical problems. In BVP a partial differential equation is constructed and solved based on existing values of the desired function on a specific surface, which are called boundary values. The solution to a BVP can be presented by an integral formula in spatial domain. The gradiometric BVPs are the special cases of BVPs in which the second-order partial derivatives of the desired function are given on the boundary. The solutions of the gradiometric BVPs are presented by three integral formulas as well. If we assume that the boundary is a sphere, then the integration should be performed on its surface. However, if the integration domain is a small part of this surface, the function will not be recovered correctly and the solution will be biased. We should look for a method to localize the integrals and reduce this bias.

The gradiometric BVPs are of major importance in satellite gravity gradiometry. The second-order partial derivatives of the geopotential have more sensitivity to the Earth gravitational field than the other existing space techniques and the geopotential coefficients can be recovered to high degrees and orders, say to 300; see for example Rummel et al. (1993), Koop (1993), Balmino et al. (1998) and (2001) and Albertella et al. (2002). In the gradiometric BVP three combinations for the partial secondorder derivatives of geopotential are constructed so that tensor spherical harmonics (SHs) can be used to make some integral formulas for recovering the gravity field; 
see for instance Rummel (1997), van Gelderen and Rummel (2001) and (2002) and Martinec (2003).

Grünbaum et al. (1982) discussed the problem of finite convolution integral operator. Spatial localization is an issue for analyzing the satellite altimetry data lacking a full global coverage. Mainville (1986) was one of the earliest persons who presented recursive formulas for the integral product of the associated Legendre functions (ALFs) and used them in processing of the satellite altimetry data. Later on Hwang (1991) presented another recursive formula for generating the Gaunt coefficients (Varshalovich et al. 1989) and used them for evaluating of this integral product and solving a similar problem. Simons et al. (1997) localized the gravity field of the Venus for studying its tectonic dynamics. Pail et al. (2001) also used some numerical techniques to orthonormalize the base functions or SHs having a global orthogonality support for local gravity field determination. Another idea which is of interest today is the Slepian method (Slepian 1983). In this method the ratio of the signal energy in the local and global senses is analyzed. The eigenvectors which maximize this ratio are selected as the orthogonal base functions with a local concentration. Slepian (1983) presented the method for one-dimensional signals but later it was generalized to two-dimensions by Albertella et al. (1999), Miranian (2004) and Wiezorek and Simons (2005) and further developed by Simons et al. (2006).

Until now most of the studies are related to a spatially restricted integral (SRI) product of scalar SHs. However, having such a product is not sufficient for localizing the solutions of the gradiometric BVPs. The spherical Slepian functions in these BVPs involve the integral products of the vector and tensor SHs. These integral products are spatially restricted as well. It means that the integration domain is not a full sphere. This paper will simplify these SRIs in terms of integral of ALFs and combinations of the Gaunt coefficients.

\section{THE GRADIOMETRIC BOUNDARY VALUE PROBLEMS}

Let $V(P)$ be a harmonic function satisfying the Laplace partial differential equation $\Delta V(P)=0$, where $\Delta$ stands for the Laplacian operator (Heiskanen and Moritz 1967 , p. 5). Solution of this partial differential equation outside a sphere with radius $R$ is:

$$
V(P)=\sum_{n=0}^{\infty}\left(\frac{R}{r}\right)^{n+1} \sum_{m=-n}^{n} v_{n m} Y_{n m}(P),
$$

where $r$ is the geocentric distance of the point $P, v_{n m}$ is the SH coefficients of degree $n$ and order $m$,

$$
Y_{n m}(P)=e^{i m \lambda} P_{n m}
$$


is the surface $\mathrm{SH}, P_{n m}=P_{n m}(\cos \theta)$ is the ALF of degree $n$ and order $m$ and $\theta$ and $\lambda$ are the co-latitude and longitude of $P$, respectively. Finally $e$ stands for the exponential function.

Now we assume that instead of $V(P)$ we have its second-order partial derivatives on a sphere with the radius $r$. Here we use the local north-oriented reference frame for expressing these derivatives. This frame is defined as a frame whose $z$-axis is pointing upwards in geocentric radial direction, $x$-axis towards the north and the frame is right handed, it means that $y$-axis is directed to the west. The derivatives can construct the well-known gradiometric tensor:

$$
\mathbf{V}(P)=\left[\begin{array}{ccc}
V_{x x}(P) & V_{x y}(P) & V_{x z}(P) \\
V_{y x}(P) & V_{y y}(P) & V_{y z}(P) \\
V_{z x}(P) & V_{z y}(P) & V_{z z}(P)
\end{array}\right] .
$$

This tensor can be decomposed into symmetric and anti-symmetric parts:

$$
\begin{aligned}
\mathbf{V}(P) & =V_{z z}(P) \mathbf{e}_{z z}+2 V_{x z}(P) \mathbf{e}_{x z}+2 V_{y z}(P) \mathbf{e}_{y z}+\frac{1}{2}\left[V_{x x}(P)-V_{y y}(P)\right]\left(\mathbf{e}_{x x}-\mathbf{e}_{y y}\right)+ \\
& +2 V_{x y}(P) \mathbf{e}_{x y}-\frac{1}{2}\left[V_{x x}(P)+V_{y y}(P)\right]\left(\mathbf{e}_{x x}+\mathbf{e}_{y y}\right),
\end{aligned}
$$

where $\mathbf{e}_{i j}=\left(\mathbf{e}_{i} \otimes \mathbf{e}_{j}\right), i, j=x, y, z$ is the symmetric spherical dyad. If we just consider the symmetric part of Eq. (1d) which has similar expressions with tensor SHs, we can write (van Gelderen and Rummel 2001, Martinec 2003, Eshagh 2009a, 2009b and 2010):

$$
\begin{aligned}
& V_{z z}(P) \mathbf{e}_{z z}=\frac{1}{R^{2}} \sum_{n=0}^{\infty}(n+1)(n+2)\left(\frac{R}{r}\right)^{n+3} \sum_{m=-n}^{n} v_{n m} \mathbf{Z}_{n m}^{(1)}(P), \\
& V_{x z}(P) \mathbf{e}_{x z}+V_{y z}(P) \mathbf{e}_{y z}=\frac{1}{R^{2}} \sum_{n=1}^{\infty}(n+2)\left(\frac{R}{r}\right)^{n+3} \sum_{m=-n}^{n} v_{n m} \mathbf{Z}_{n m}^{(2)}(P), \\
& \left(V_{x x}(P)-V_{y y}(P)\right)\left(\mathbf{e}_{x x}-\mathbf{e}_{y y}\right)+2 V_{x y}(P) \mathbf{e}_{x y}=\frac{1}{R^{2}} \sum_{n=2}^{\infty}\left(\frac{R}{r}\right)^{n+3} \sum_{m=-n}^{n} v_{n m} \mathbf{Z}_{n m}^{(3)}(P) .
\end{aligned}
$$

where (Zerilli 1970) :

$$
\begin{aligned}
& \mathbf{Z}_{n m}^{(1)}(P)=Y_{n m}(P) \mathbf{e}_{z z}, \\
& \mathbf{Z}_{n m}^{(2)}(P)=E_{n m}(P) \mathbf{e}_{x z}+F_{n m}(P) \mathbf{e}_{y z}, \\
& \mathbf{Z}_{n m}^{(3)}(P)=G_{n m}(P)\left(\mathbf{e}_{x x}-\mathbf{e}_{y y}\right)+2 H_{n m}(P) \mathbf{e}_{x y} .
\end{aligned}
$$


In Equations (2a)-(2c) $\mathbf{Z}_{n m}^{(1)}(P), \mathbf{Z}_{n m}^{(2)}(P)$ and $\mathbf{Z}_{n m}^{(3)}(P)$ are the tensor SHs. In order to clarify their definitions see Appendix A. Eshagh (2009a, 2009b, 2009c and 2010) presented the functions $E_{n m}(P), F_{n m}(P), G_{n m}(P)$ and $H_{n m}(P)$ in the following forms:

$$
\begin{aligned}
& E_{n m}(P)=e^{i m \lambda} \frac{\partial P_{n m}}{\partial \theta}=e^{i m \lambda}\left\{a_{n m}^{1} P_{n, m-1}+a_{n m}^{2} P_{n, m+1}\right\}, \\
& F_{n m}(P)=i m e^{i m \lambda} \frac{P_{n m}}{\sin \theta}=i e^{i m \lambda} \begin{cases}b_{n m}^{1} P_{n-1, m-1}+b_{n m}^{2} P_{n-1, m+1} & \text { (backward mode) } \\
b_{n m}^{\prime 1} P_{n+1, m-1}+b_{n m}^{\prime 2} P_{n+1, m+1} & \text { (forward mode) }\end{cases} \\
& G_{n m}(P)=e^{i m \lambda}\left(\frac{\partial^{2} P_{n m}}{\partial \theta^{2}}-\cot \theta \frac{\partial P_{n m}}{\partial \theta}+m^{2} \frac{P_{n m}}{\sin ^{2} \theta}\right)= \\
& =e^{i m \lambda}\left\{c_{n m}^{1} P_{n, m-2}+c_{n m}^{2} P_{n m}+c_{n m}^{3} P_{n, m+2}\right\}, \\
& H_{n m}(P)=2 i m e^{i m \lambda} \frac{\partial}{\partial \theta}\left(\frac{P_{n m}}{\sin \theta}\right)= \\
& =i e^{i m \lambda}\left\{\begin{array}{ll}
d_{n m}^{1} P_{n-1, m-2}+d_{n m}^{2} P_{n-1, m}+d_{n m}^{3} P_{n-1, m+2} & \text { (backward mode) } \\
d_{n m}^{\prime \prime} P_{n+1, m-2}+d_{n m}^{\prime 2} P_{n+1, m}+d_{n m}^{\prime 3} P_{n+1, m+2} & \text { (forward mode) }
\end{array} .\right.
\end{aligned}
$$

The constant coefficients $a_{n m}^{1}, a_{n m}^{2}, b_{n m}^{1}, b_{n m}^{2}, b_{n m}^{\prime 1}, b_{n m}^{\prime 2}$ will be presented in Lemmas 1, 2 and 3. $c_{n m}^{1}, c_{n m}^{2}, c_{n m}^{3}, d_{n m}^{1}, d_{n m}^{2}, d_{n m}^{3}, d_{n m}^{\prime 1}, d_{n m}^{\prime 2}$ and $d_{n m}^{\prime 3}$ are $(2009 \mathrm{~b}$ and 2010):

$$
\begin{aligned}
& c_{n m}^{1}=\frac{1}{2}(n+m)(n-m+1)(n+m-1)(n-m+2), \\
& c_{n m}^{2}=m^{2}, \\
& d_{n m}^{1}=\frac{1}{2}(n-m+1)(n-m+2)(n+m)(n-m+3), \\
& d_{n m}^{2}=m(n-m+1), \\
& d_{n m}^{\prime 1}=\frac{1}{2}(n+m)(n+m-1)(n+m-2)(n-m+1), \\
& d_{n m}^{\prime 2}=-m(n+m), \\
& d_{n m}^{3}=d_{n m}^{\prime 3}=c_{n m}^{3}=\frac{1}{2} .
\end{aligned}
$$

The fully-normalized versions of these coefficients were given by Eshagh (2008, 2009a and 2010). In Eqs. (4b) and (4d) two different formulas were presented. The formulas involving $n-1$ are called backward mode because the degree of the function in the left hand side of the equations is $n$. We call the other formulas involving $n+1$ forward mode. 


\section{SPHERICAL SLEPIAN FUNCTION FOR GRADIOMETRIC BOUNDARY VALUE PROBLEMS}

The idea of Slepian (1983) was to maximize the energy of a signal inside a specific region using the following ratio:

$$
s=\frac{\|V\|_{\sigma_{0}}^{2}}{\|V\|_{\sigma}^{2}}=\frac{\iint_{\sigma_{0}} V^{2}(Q) d \sigma}{\iint_{\sigma} V^{2}(Q) d \sigma}, \quad \sigma_{0} \subset \sigma
$$

where $\|\bullet\|_{\sigma}^{2}$ and $\|\bullet\|_{\sigma_{0}}^{2}$ are the definition of squared-norms on the surfaces $\sigma$ and $\sigma_{0}$, respectively; $Q$ stands for integration point and $d \sigma$ is the integration element. In the spherical domain, these norms are the spatial globalaverage of the squared functions. We should find in which condition the ratio (6) is maximized and the signal power is maximal in $\sigma_{0}$. In order to explain this matter, let Eq. (1a) be the SH expansion of the signal in $\sigma$. Similar to Eq. (6) we obtain the spherical Slepian function based on Eq. (1a):

$$
S=\frac{\sum_{n=0}^{\infty}\left(\frac{R}{r}\right)^{n+1} \sum_{m=-n}^{n} v_{n m} \sum_{n^{\prime}=0}^{\infty}\left(\frac{R}{r}\right)^{n^{\prime}+1} \sum_{m^{\prime}=-n^{\prime}}^{n^{\prime}} v_{n^{\prime} m^{\prime}} \iint_{\sigma_{0}} Y_{n m}(Q) Y_{n^{\prime} m^{\prime}}^{*}(Q) d \sigma}{\sum_{n=0}^{\infty}\left(\frac{R}{r}\right)^{n+1} \sum_{m=-n}^{n} v_{n m} \sum_{n^{\prime}=0}^{\infty}\left(\frac{R}{r}\right)^{n^{\prime}+1} \sum_{m^{\prime}=-n^{\prime}}^{n^{\prime}} v_{n^{\prime} m^{\prime}} \iint_{\sigma} Y_{n m}(Q) Y_{n^{\prime} m^{\prime}}^{*}(Q) d \sigma},
$$

where $*$ stands for the complex conjugate operator. The integral in the denominator of Eq. (7a) is being taken on $\sigma$ while the one in the numerator is spatially restricted to $\sigma_{0}$. According to the orthogonality property of SHs (see Appendix A) the former case is simplified and Eq. (7a) will change to:

$$
S=\frac{\sum_{n=0}^{\infty}\left(\frac{R}{r}\right)^{n+1} \sum_{m=-n}^{n} v_{n m} \sum_{n^{\prime}=0}^{\infty}\left(\frac{R}{r}\right)^{n^{\prime}+1} \sum_{m^{\prime}=-n^{\prime}}^{n^{\prime}} v_{n^{\prime} m^{\prime}} D_{n m n^{\prime} m^{\prime}}}{4 \pi \sum_{n=0}^{\infty}\left(\frac{R}{r}\right)^{2 n+2} \sum_{m=-n}^{n} v_{n m}^{2}},
$$

where

$$
D_{n m n^{\prime} m^{\prime}}=\iint_{\sigma_{0}} Y_{n m}(Q) Y_{n^{\prime} m^{\prime}}^{*}(Q) d \sigma
$$

Since the SHs are just globally orthogonal when we restrict the integral domain to $\sigma_{0}$ this property is destroyed. If we write Eq. (7c) into a matrix form we have (see also Kim and Tapley 2000): 


$$
\mathbf{D}=\left[\begin{array}{ccc}
D_{00,00} & \cdots & D_{00, N N} \\
\vdots & \ddots & \vdots \\
D_{N N, 00} & \cdots & D_{N N, N N}
\end{array}\right]
$$

It is straightforward to obtain the eigenvalues and eigenvectors of this matrix. We select those eigenvalues and eigenvectors which maximize Eq. (7b) as the base functions for spatial localization of the signal. In a very similar way, we can find the spherical Slepian functions corresponding to Eqs. (2a)-(2c) which are harmonic expressions of combinations of gradiometric tensor elements in terms of tensor SHs. The Slepian function based on Eq. (2a) which involves scalar SHs is:

$$
s=\frac{\sum_{n=0}^{\infty}(n+1)(n+2)\left(\frac{R}{r}\right)^{n+3} \sum_{m=-n}^{n} v_{n m} \sum_{n^{\prime}=0}^{\infty}\left(n^{\prime}+1\right)\left(n^{\prime}+2\right)\left(\frac{R}{r}\right)^{n^{\prime}+3} \sum_{m^{\prime}=-n^{\prime}}^{n^{\prime}} v_{n^{\prime} m^{\prime}} \iint_{\sigma_{0}} \mathbf{Z}_{n m}^{(1)}(Q):\left[\mathbf{Z}_{n^{\prime} m^{\prime}}^{(1)}(Q)\right]^{*} d \sigma}{4 \pi \sum_{n=0}^{\infty}(n+1)^{2}(n+2)^{2}\left(\frac{R}{r}\right)^{2 n+6} \sum_{m=-n}^{n} v_{n m}^{2}}
$$

A spherical Slepian function can be found based on Eq. (2b) based on vector SHs:

$$
s=\frac{\sum_{n=1}^{\infty}(n+2)\left(\frac{R}{r}\right)^{n+3} \sum_{m=-n}^{n} v_{n m} \sum_{n^{\prime}=1}^{\infty}\left(n^{\prime}+2\right)\left(\frac{R}{r}\right)^{n^{\prime}+3} \sum_{m^{\prime}=-n^{\prime}}^{n^{\prime}} v_{n^{\prime} m^{\prime}} \int_{\sigma_{0}} \mathbf{Z}_{n m}^{(2)}(Q):\left[\mathbf{Z}_{n^{\prime} m^{\prime}}^{(2)}(Q)\right]^{*} d \sigma}{4 \pi \sum_{n=1}^{\infty} n(n+1)(n+2)^{2}\left(\frac{R}{r}\right)^{2 n+6} \sum_{m=-n}^{n} v_{n m}^{2}}
$$

and for Eq. (2c) the Slepian function will be:

$$
S=\frac{\sum_{n=2}^{\infty}\left(\frac{R}{r}\right)^{n+3} \sum_{m=-n}^{n} v_{n m} \sum_{n^{\prime}=2}^{\infty}\left(\frac{R}{r}\right)^{n^{\prime}+3} \sum_{m^{\prime}=-n^{\prime}}^{n^{\prime}} v_{n^{\prime} m^{\prime}} \iint_{\sigma_{0}} \mathbf{Z}_{n m}^{(3)}(Q):\left[\mathbf{Z}_{n^{\prime} m^{\prime}}^{(3)}(Q)\right]^{*} d \sigma}{4 \pi \sum_{n=2}^{\infty}(n-1) n(n+1)(n+2)\left(\frac{R}{r}\right)^{2 n+6} \sum_{m=-n}^{n} v_{n m}^{2}} .
$$

The denominators of Eqs. (8b) and (8c) are obtained by taking the global average of Eqs. (2b) and (2c) considering the norm of tensor SHs; see Appendix A. Three SRIs are seen in the numerators of Eqs. (8a)-(8c). The surface integral of Eq. (8a) is the SRI product of two scalar SHs. Equations (8b) and (8c) involve the SRI products of two vector and two tensor SHs, respectively. The solutions of these SRIs are the most important step of spatially localization of the gravitational signal in the gradiometric BVPs.

\section{THE SPATIALLY RESTRICTED INTEGRALS}

In this section we formulate the SRI products of the scalar, vector and tensor SHs. To do that, the following lemmas are introduced and used in our mathematical derivations. 
Lemma 1 (Ilk 1983, Eq. Z.1.44):

where

$$
\frac{\partial P_{n m}}{\partial \theta}=a_{n m}^{1} P_{n, m-1}+a_{n m}^{2} P_{n, m+1},
$$

$$
a_{n m}^{1}=\frac{(n+m)(n-m+1)}{2} \text { and } a_{n m}^{2}=-\frac{1}{2} .
$$

Lemma 2 (Ilk 1983, Eq. Z.1.41):

where

$$
m \frac{P_{n m}}{\sin \theta}=b_{n m}^{1} P_{n-1, m-1}+b_{n m}^{2} P_{n-1, m+1},
$$

$$
b_{n m}^{1}=\frac{(n+m)(n+m-1)}{2} \text { and } b_{n m}^{2}=\frac{1}{2} . \quad m \neq 0
$$

Note: if $m=0, b_{n m}^{1}=b_{n m}^{2}=0$.

Lemma 3 (Ilk 1983, Eq. Z.1.42):

$$
m \frac{P_{n m}}{\sin \theta}=b_{n m}^{\prime 1} P_{n+1, m-1}+b_{n m}^{\prime 2} P_{n+1, m+1}, m \neq 0
$$

where

$$
b_{n m}^{\prime 1}=\frac{(n-m+1)(n-m+2)}{2} \text { and } b_{n m}^{\prime 2}=\frac{1}{2} .
$$

Note: if $m=0, b_{n m}^{\prime 1}=b_{n m}^{\prime 2}=0$.

\subsection{SPATIALLY RESTRICTED INTEGRAL OF TWO SCALAR SPHERICAL HARMONICS}

The scalar SHs are special cases of tensor SHs and the SRI of two scalar SHs. Based on Eq. (1b), is:

$$
\iint_{\sigma_{0}} \mathbf{Z}_{n^{\prime} m^{\prime}}^{(1)}(Q):\left[\mathbf{Z}_{n^{\prime} m^{\prime \prime}}^{(1)}(Q)\right]^{*} d \sigma=\int_{\lambda_{1}}^{\lambda 2} e^{i \Delta m \lambda} d \lambda \int_{\theta_{1}}^{\theta_{2}} P_{n^{\prime} m^{\prime}} P_{n^{\prime \prime} m^{\prime \prime}} \sin \theta d \theta,
$$

where $\Delta m=m^{\prime}-m^{\prime \prime}$.

The performance of integration with respect to $\lambda$ is easy. The solution of the second integral which is a SRI product of two ALFs needs more consideration. The SRI (9) can be performed in an arbitrary geometry of $\sigma_{0}$ by dividing the integration domain into small elements. The product of two ALFs can be written in terms of one ALF and the Gaunt coefficients (see e.g. Xu 1996): 


$$
P_{n^{\prime} m^{\prime}} P_{n^{\prime \prime} m^{\prime \prime}}=\sum_{n=\left|n^{\prime}-n^{\prime \prime}\right|}^{n^{\prime}+n^{\prime \prime}} Q_{n^{\prime} m^{\prime} n^{\prime \prime} m^{\prime \prime}}^{n P_{n m}}
$$

The Gaunt coefficients $Q_{n^{\prime} m^{\prime \prime} n^{\prime \prime} m^{\prime \prime}}^{n}$ are non-zero if $\left|n^{\prime}-n^{\prime \prime}\right|<n<n^{\prime}+n^{\prime \prime}$ and $m=m^{\prime}+m^{\prime \prime}$. There is a challenge among mathematicians to find a relevant algorithm to speed up the Gaunt coefficients generation; see for example Xu (1996) and Sebilleau (1998). A brief definition of the Gaunt coefficients are given in Appendix B.

Substituting Eq. (10) into Eq. (9) reads:

$$
\iint_{\sigma_{0}} \mathbf{Z}_{n^{\prime} m^{\prime}}^{(1)}(Q):\left[\mathbf{Z}_{n^{\prime \prime} m^{\prime \prime}}^{(1)}(Q)\right]^{*} d \sigma=\frac{i\left(e^{i \Delta m \lambda_{1}}-e^{i \Delta m \lambda_{2}}\right)}{\Delta m} \sum_{n=n^{\prime}-n^{n} \mid}^{n^{\prime}+n^{n}} Q_{n^{\prime} m^{\prime} n^{\prime} m^{\prime \prime}}^{\theta_{2}} \int_{\theta_{1}}^{P_{1}} P_{n m} \sin \theta d \theta .
$$

The solution of the integral of ALFs which is in the right hand side of Eq. (11) is rather easy and a recursive formula was presented by Paul (1978) for that

\subsection{SPATIALLY RESTRICTED INTEGRAL OF TWO VECTOR SPHERICAL HARMONICS}

The Slepian function of Eq. (8b) involves the integral products of two derivatives of ALFs and two ALFs divided by $\sin ^{2} \theta$. In this subsection we prepare some necessary formulas in Propositions 1 and 2 and after that we will formulate the SRI of vector SHs.

\section{Proposition 1}

$$
\frac{\partial P_{n^{\prime} m^{\prime}}}{\partial \theta} \frac{\partial P_{n^{\prime \prime} m^{\prime \prime}}}{\partial \theta}=\sum_{n=n^{\prime}-n^{n} \mid}^{n^{\prime}+n^{n}} \mathrm{~K}_{n^{\prime} m^{\prime} n^{\prime \prime} m^{\prime \prime}}^{n m} P_{n m}
$$

where

$$
\begin{aligned}
& \left|n^{\prime}-n^{\prime \prime}\right|<n<n^{\prime}+n^{\prime \prime}
\end{aligned}
$$

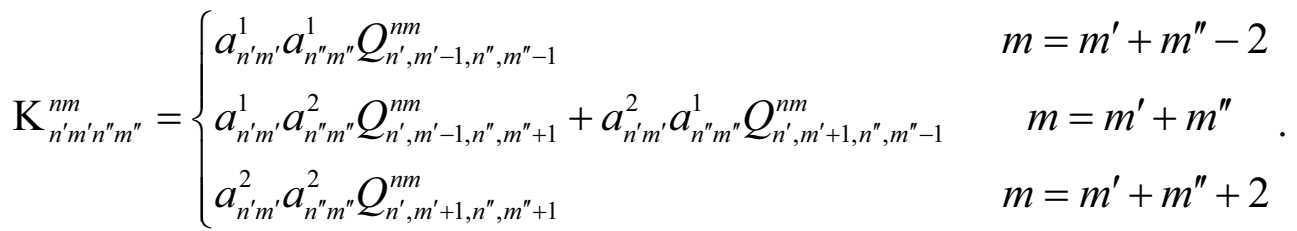

Proof. According to Lemma 1 we can write

$$
\frac{\partial P_{n^{\prime} m^{\prime}}}{\partial \theta} \frac{\partial P_{n^{\prime \prime} m^{\prime \prime}}}{\partial \theta}=\left(a_{n^{\prime} m^{\prime}}^{1} P_{n^{\prime}, m^{\prime}-1}+a_{n^{\prime} m^{\prime}}^{2} P_{n^{\prime}, m^{\prime}+1}\right)\left(a_{n^{\prime \prime} m^{\prime \prime}}^{1} P_{n^{\prime \prime}, m^{\prime \prime}-1}+a_{n^{\prime \prime} m^{\prime \prime}}^{2} P_{n^{\prime \prime}, m^{\prime \prime}+1}\right),
$$

Expanding the right hand side of Eq. (12a) considering Eq. (10) yields: 


$$
\begin{aligned}
& \frac{\partial P_{n^{\prime} m^{\prime}}}{\partial \theta} \frac{\partial P_{n^{\prime \prime} m^{n}}}{\partial \theta}=a_{n^{\prime} m^{\prime}}^{1} a_{n^{\prime \prime} m^{\prime \prime}}^{1} \sum_{n=n n^{\prime}-n^{\prime \prime}}^{n^{\prime}+n^{n}} Q_{n^{\prime}, m^{\prime}-1, n^{\prime \prime}, m^{\prime \prime}-1}^{n P_{n m}}+ \\
& +a_{n^{\prime} m^{\prime}}^{1} a_{n^{\prime \prime} m^{\prime \prime}}^{2} \sum_{n=\left|n^{\prime}-n^{\prime \prime}\right|}^{n^{\prime}+n^{\prime \prime}} Q_{n^{\prime}, m^{\prime}-1, n^{\prime \prime}, m^{\prime \prime}+1}^{n P_{n m}}+a_{n^{\prime} m^{\prime}}^{2} a_{n^{\prime \prime} m^{\prime \prime}}^{1} \sum_{n=n^{\prime}-n^{\prime \prime} \mid}^{n^{\prime}+n^{\prime \prime}} Q_{n^{\prime}, m^{\prime}+1, n^{\prime \prime}, m^{\prime \prime}-1}^{n m} P_{n m}+ \\
& +a_{n^{\prime} m}^{2} a_{n^{\prime \prime} m^{\prime \prime}}^{2} \sum_{n=\left|n^{\prime}-n^{\prime \prime}\right|}^{n^{\prime}+n^{\prime \prime}} Q_{n^{\prime}, m^{\prime}+1, n^{\prime \prime}, m^{\prime \prime}+1}^{n} P_{n m} \text {. }
\end{aligned}
$$

After simplifications we obtain:

$$
\begin{aligned}
\frac{\partial P_{n^{\prime} m^{\prime}}}{\partial \theta} \frac{\partial P_{n^{\prime \prime} m^{\prime \prime}}}{\partial \theta} & =\sum_{n=n^{\prime} n^{\prime \prime} \mid}^{n^{\prime}+n^{n}}\left(a_{n^{\prime} m^{\prime}}^{1} a_{n^{\prime \prime} m^{\prime \prime}}^{1} Q_{n^{\prime}, m^{\prime}-1, n^{\prime \prime}, m^{\prime \prime}-1}^{n m}+a_{n^{\prime} m^{\prime}}^{1} a_{n^{\prime \prime} m^{\prime \prime}}^{2} Q_{n^{\prime}, m^{\prime}-1, n^{\prime \prime}, m^{\prime \prime}+1}^{n m}+\right. \\
& \left.+a_{n^{\prime} m^{\prime}}^{2} \cdot a_{n^{\prime \prime} m^{\prime}}^{1} Q_{n^{\prime}, m^{\prime}+1, n^{\prime \prime}, m^{\prime \prime}-1}^{n+1}+a_{n^{\prime} m^{\prime}}^{2} \cdot a_{n^{\prime \prime} m^{\prime}}^{2} Q_{n^{\prime}, m^{\prime}+1, n^{\prime \prime}, m^{\prime \prime}+1}^{n m}\right) P_{n m} .
\end{aligned}
$$

The Gaunt coefficients are non-zero for the orders which are equal to the sum of the orders of the first and second ALFs. Therefore if in the right hand side of Eq. (12c) the Gaunt coefficients of the first term are non-zero then those of other terms are. This holds for the last term as well. If the second and third terms are non-zero then the first and last terms will be zero. Consequently it is convenient to write Eq. (12c) as the equation presented in the proposition.

\section{Proposition 2}

where

$$
\frac{P_{n^{\prime} m^{\prime}}}{\sin \theta} \frac{P_{n^{\prime \prime} m^{\prime \prime}}}{\sin \theta}=\sum_{n=n^{\prime} n^{n}-2 \mid}^{n^{\prime}+n^{n}+2}\left(\Gamma^{v}\right)_{n^{\prime} m^{\prime} n^{\prime \prime} m^{\prime \prime}}^{n m} P_{n m}, v=1,2,3 \text { or } 4
$$

$$
\left(\Gamma^{1}\right)_{n^{\prime} m^{\prime} n^{\prime \prime} m^{\prime \prime}}^{n m}= \begin{cases}b^{\prime}-n^{\prime \prime} \mid<n<n^{\prime}+n^{\prime \prime}-2 & m=m^{\prime}+m^{\prime \prime}-2 \\ b_{n^{\prime} m}^{1}, b_{n^{\prime \prime} m^{\prime \prime}}^{1} Q_{n^{\prime}-1, m^{\prime}-1, n^{\prime \prime}-1, m^{\prime \prime}-1}^{n} b_{n^{\prime \prime} m^{\prime \prime}}^{n} Q_{n^{\prime}-1, m^{\prime}-1, n^{\prime \prime}-1, m^{\prime \prime}+1}^{n+}+b_{n^{\prime} m^{\prime}}^{2} b_{n^{\prime \prime} m^{\prime \prime}}^{1} Q_{n^{\prime}-1, m^{\prime}+1, n^{\prime \prime}-1, m^{\prime \prime}-1}^{n m} & m=m^{\prime}+m^{\prime \prime}, \\ b_{n^{\prime} m^{\prime}}^{2}, b_{n^{\prime \prime} m^{\prime \prime}}^{2} Q_{n^{\prime}-1, m^{\prime}+1, n^{\prime \prime}-1, m^{\prime \prime}+1}^{n m} & m=m^{\prime}+m^{\prime \prime}+2\end{cases}
$$

or

$$
\left(\Gamma^{2}\right)_{n^{\prime} m^{\prime} n^{\prime \prime} m^{\prime \prime}}^{n m}= \begin{cases}n^{\prime}-n^{\prime \prime}+2 \mid<n<n^{\prime}+n^{\prime \prime} & m=m^{\prime}+m^{\prime \prime}-2 \\ b_{n^{\prime} m}^{\prime \prime}, b_{n^{\prime \prime} m^{\prime \prime}}^{1} Q_{n^{\prime}+1, m^{\prime}-1, n^{\prime \prime}-1, m^{\prime \prime}-1}^{n m} & m=m^{\prime}+m^{\prime \prime}, \\ b_{n^{\prime} m}^{\prime 1}, b_{n^{\prime \prime} m^{\prime \prime}}^{2} Q_{n^{\prime}+1, m^{\prime}-1, n^{\prime \prime}-1, m^{\prime \prime}+1}^{n m}+b_{n^{\prime} m^{\prime}}^{\prime 2} b_{n^{\prime \prime} m^{\prime \prime}}^{1} Q_{n^{\prime}+1, m^{\prime}+1, n^{\prime \prime}-1, m^{\prime \prime}-1}^{n m} & m=m^{\prime}+m^{\prime \prime}+2 \\ b_{n^{\prime} m^{\prime}}^{\prime 2}, b_{n^{\prime \prime} m^{\prime \prime}}^{2} Q_{n^{\prime}+1, m^{\prime}+1, n^{\prime \prime}-1, m^{\prime \prime}+1}^{n m} & m\end{cases}
$$

or

$$
\left|n^{\prime}-n^{\prime \prime}-2\right|<n<n^{\prime}+n^{\prime \prime}
$$




$$
\left(\Gamma^{3}\right)_{n^{\prime} m^{\prime} n^{\prime \prime} m^{\prime \prime}}^{n m}=\left\{\begin{array}{ll}
b_{n^{\prime} m^{\prime}}^{1}, b_{n^{\prime \prime} m^{\prime \prime}}^{\prime 1} Q_{n^{\prime}-1, m^{\prime}-1, n^{\prime \prime}+1, m^{\prime \prime}-1}^{n m} & m=m^{\prime}+m^{\prime \prime}-2 \\
b_{n^{\prime} m^{\prime}}^{1}, b_{n^{\prime \prime} m^{\prime \prime}}^{\prime 2} Q_{n^{\prime}-1, m^{\prime}-1, n^{\prime \prime}+1, m^{\prime \prime}+1}^{n m}+b_{n^{\prime} m^{\prime}}^{2}, b_{n^{\prime \prime} m^{\prime \prime}}^{\prime 1} Q_{n^{\prime}-1, m^{\prime}-1, n^{\prime \prime}+1, m^{\prime \prime}-1}^{n m} & m=m^{\prime}+m^{\prime \prime} \\
b_{n^{\prime} m^{\prime}}^{2}, b_{n^{\prime \prime} m^{\prime \prime}}^{\prime 2} Q_{n^{\prime}-1, m^{\prime}+1, n^{\prime \prime}+1, m^{\prime \prime}+1}^{n m} & m=m^{\prime}+m^{\prime \prime}+2
\end{array},\right.
$$

or

$$
\left(\Gamma^{4}\right)_{n^{\prime} m^{\prime} n^{\prime \prime} m^{\prime \prime}}^{n m}=\left\{\begin{array}{ll}
b_{n^{\prime} m^{\prime}}^{\prime 1}, b_{n^{\prime \prime} m^{\prime \prime}}^{\prime 1} Q_{n^{\prime}+1, m^{\prime}-1, n^{\prime \prime}+1, m^{\prime \prime}-1}^{n m} & m=m^{\prime}+m^{\prime \prime}-2 \\
b_{n^{\prime} m^{\prime}}^{\prime 1}, b_{n^{\prime \prime} m^{\prime \prime}}^{\prime 2} Q_{n^{\prime}+1, m^{\prime}-1, n^{\prime \prime}+1, m^{\prime \prime}+1}^{n m}+b_{n^{\prime} m^{\prime}}^{\prime 2}, b_{n^{\prime \prime} m^{\prime \prime}}^{\prime 1} Q_{n^{\prime}+1, m^{\prime}+1, n^{\prime \prime}+1, m^{\prime \prime}-1}^{n m} & m=m^{\prime}+m^{\prime \prime} \\
b_{n^{\prime} m^{\prime}}^{\prime 2}, b_{n^{\prime \prime} m^{\prime \prime}}^{\prime 2} Q_{n^{\prime}+1, m^{\prime}+1, n^{\prime \prime}+1, m^{\prime \prime}+1}^{n m} & m=m^{\prime}+m^{\prime \prime}+2
\end{array} .\right.
$$

Proof. According to Lemma 2 we can write

$$
\frac{P_{n^{\prime} m^{\prime}}}{\sin \theta} \frac{P_{n^{\prime \prime} m^{\prime \prime}}}{\sin \theta}=\left(b_{n^{\prime} m^{\prime}}^{1} P_{n^{\prime}-1, m^{\prime}-1}+b_{n^{\prime} m^{\prime}}^{2} P_{n^{\prime}-1, m^{\prime}+1}\right)\left(b_{n^{\prime \prime} m^{\prime \prime}}^{1} P_{n^{\prime \prime}-1, m^{\prime \prime}-1}+b_{n^{\prime \prime} m^{\prime \prime}}^{2} P_{n^{\prime \prime}-1, m^{\prime \prime}+1}\right),
$$

after expanding Eq. (13a) considering Eq. (10) we obtain:

$$
\begin{aligned}
\frac{P_{n^{\prime} m^{\prime}}}{\sin \theta} & \frac{P_{n^{\prime \prime} m^{\prime \prime}}}{\sin \theta}=b_{n^{\prime} m^{\prime}}^{1}, b_{n^{\prime \prime} m^{\prime \prime}}^{1} \sum_{n=\left|n^{\prime} n^{\prime \prime}\right|}^{n^{\prime}+n^{\prime \prime}-2} Q_{n^{\prime}-1, m^{\prime}-1, n^{\prime \prime}-1, m^{\prime \prime}-1}^{n m} P_{n m}+ \\
& +b_{n^{\prime} m^{\prime}}^{1}, b_{n^{\prime \prime} m^{\prime \prime}}^{2} \sum_{n=\left|n^{\prime}-n^{\prime \prime}-2\right|}^{n^{\prime}+n^{\prime \prime}} Q_{n^{\prime}-1, m^{\prime}-1, n^{\prime \prime}+1, m^{\prime \prime}+1}^{n m} P_{n m}+b_{n^{\prime} m^{\prime}}^{2}, b_{n^{\prime \prime} m^{\prime \prime}}^{1} \sum_{n=\left|n^{\prime}-n^{\prime \prime}+2\right|}^{n^{\prime}+n^{\prime \prime}} Q_{n^{\prime}+1, m^{\prime}+1, n^{\prime \prime}-1, m^{\prime \prime}-1}^{n m} P_{n m}+ \\
& +b_{n^{\prime} m^{\prime}}^{2}, b_{n^{\prime \prime} m^{\prime \prime}}^{2} \sum_{n=\left|n^{\prime}-n^{\prime \prime}\right|}^{n^{\prime}+n^{\prime \prime}+2} Q_{n^{\prime}+1, m^{\prime}+1, n^{\prime \prime}+1, m^{\prime \prime}+1}^{n m} P_{n m} .
\end{aligned}
$$

Finally we obtain:

$$
\begin{aligned}
\frac{P_{n^{\prime} m^{\prime}}}{\sin \theta} \frac{P_{n^{\prime \prime} m^{\prime \prime}}}{\sin \theta}=\sum_{n=\left|n^{\prime}-n^{\prime \prime}-2\right|}^{n^{\prime}+n^{\prime \prime}+2}\left(b_{n^{\prime} m^{\prime}}^{1} b_{n^{\prime \prime} m^{\prime \prime}}^{1} Q_{n^{\prime}-1, m^{\prime}-1, n^{\prime \prime}-1, m^{\prime \prime}-1}^{n m}+b_{n^{\prime} m^{\prime}}^{1} b_{n^{\prime \prime} m^{\prime \prime}}^{2} Q_{n^{\prime}-1, m^{\prime}-1, n^{\prime \prime}+1, m^{\prime \prime}+1}^{n m}+\right. \\
\left.+b_{n^{\prime} m^{\prime}}^{2} b_{n^{\prime \prime} m^{\prime \prime}}^{1} Q_{n^{\prime}+1, m^{\prime}+1, n^{\prime \prime}-1, m^{\prime \prime}-1}^{n m}+b_{n^{\prime} m^{\prime}}^{2} b_{n^{\prime \prime} m^{\prime \prime}}^{2} Q_{n^{\prime}+1, m^{\prime}+1, n^{\prime \prime}+1, m^{\prime \prime}+1}^{n m}\right) P_{n m} .
\end{aligned}
$$

Similar expressions can be made as that presented at the end of the proof of Proposition 1. Here we just considered Lemma 2 in our derivations but other combinations can be used as well. Here we presented the final results in the proposition. If Lemmas 2 and 3 are used $\left(\Gamma^{2}\right)_{n^{\prime} m^{\prime} n^{\prime \prime} m^{\prime \prime}}^{n m}$ and $\left(\Gamma^{3}\right)_{n^{\prime} m^{\prime} n^{\prime \prime} m^{\prime \prime}}^{n m}$ will be obtained and $\left(\Gamma^{4}\right)_{n^{\prime} m^{\prime} n^{\prime \prime} m^{\prime \prime}}^{n m}$ is derived when just Lemma 3 is used.

Now, we return to formulation of the SRI of two vector SHs presented in Slepian function (8b). According to Eqs. (4a) and (4b) we can write: 


$$
\iint_{\sigma_{0}} \mathbf{Z}_{n^{\prime} m^{\prime}}^{(2)}(Q):\left[\mathbf{Z}_{n^{\prime \prime} m^{\prime \prime}}^{(2)}(Q)\right]^{*} d \sigma=\iint_{\sigma_{0}}\left(E_{n^{\prime} m^{\prime}}(Q) E_{n^{\prime \prime} m^{\prime \prime}}^{*}(Q)+F_{n^{\prime} m^{\prime}}(Q) F_{n^{\prime \prime} m^{\prime \prime}}^{*}(Q)\right) d \sigma
$$

As is observed, Eq. (14a) consists of two parts. After simplifications and integration with respect to $\lambda$, the first and second parts are:

$$
\begin{aligned}
& \iint_{\sigma_{0}} E_{n^{\prime} m^{\prime}}(Q) E_{n^{\prime \prime} m^{\prime \prime}}^{*}(Q) d \sigma=\frac{e^{i \Delta m \lambda_{2}}-e^{i \Delta m \lambda_{1}}}{i \Delta m} \int_{\theta_{1}}^{\theta_{2}} \frac{\partial P_{n^{\prime} m^{\prime}}}{\partial \theta} \frac{\partial P_{n^{\prime \prime} m^{\prime \prime}}}{\partial \theta} \sin \theta d \theta \\
& \iint_{\sigma_{0}} F_{n^{\prime} m^{\prime}}(Q) F_{n^{\prime \prime} m^{\prime \prime}}^{*}(Q) d \sigma=\frac{m^{\prime} m^{\prime \prime}\left(e^{i \Delta m \lambda_{2}}-e^{i \Delta m \lambda_{1}}\right)}{i \Delta m} \int_{\theta_{1}}^{\theta_{2}} \frac{P_{n^{\prime} m^{\prime}}}{\sin \theta} \frac{P_{n^{\prime \prime} m^{\prime \prime}}}{\sin \theta} \sin \theta d \theta .
\end{aligned}
$$

Substituting Eqs. (14c) and (14b) into Eq. (14a) with considering Propositions 1 and 2 reads:

$$
\begin{aligned}
\iint_{\sigma_{0}} \mathbf{Z}_{n^{\prime} m^{\prime}}^{(2)}(Q):\left[\mathbf{Z}_{n^{\prime \prime} m^{\prime \prime}}^{(2)}(Q)\right]^{*} d \sigma= & \frac{i\left(e^{i \Delta m \lambda_{1}}-e^{i \Delta m \lambda_{2}}\right)}{\Delta m} \sum_{n=\left|n^{\prime}-n^{\prime \prime}-2\right|}^{n^{\prime}+n^{\prime \prime}+2}\left[m^{\prime} m^{\prime \prime}\left(\Gamma^{v}\right)_{n^{\prime} m^{\prime} n^{\prime \prime} m^{\prime \prime}}^{n m}+\mathrm{K}_{n^{\prime} m^{\prime} n^{\prime \prime} m^{\prime \prime}}^{n m}\right] \times \\
& \times \int_{\theta_{1}}^{\theta_{2}} P_{n m} \sin \theta d \theta
\end{aligned}
$$

In Eq. (14d) one may say that the summation is performed from $\left|n^{\prime}-n^{\prime \prime}-2\right|$ to $n^{\prime}+n^{\prime \prime}+2$ for $\left(\Gamma^{v}\right)_{n^{\prime} m^{\prime} n^{\prime \prime} m^{\prime \prime}}^{n m}$ and it is ioonsistent $\mathrm{w}$ ith those of $\mathrm{K}_{n^{\prime} m^{\prime} n^{\prime \prime} m^{\prime \prime}}^{n m}$. However since $\mathrm{K}_{n^{\prime} m^{\prime} n^{\prime \prime} m^{\prime \prime}}^{n m}$ is non-zero when $\left|n^{\prime}-n^{\prime \prime}\right|<n<n^{\prime}+n^{\prime \prime}$ therefore one should not worry about this issue.

\subsection{SPATIALLY RESTRICTED INTEGRAL OF TWO TENSOR SPHERICAL HARMONICS}

The Slepian function of Eq. (8c) involves two complicated SRI products of two tensor SHs. Their formulations will not be as easy as those were for the vector SHs. This subsection will simplify some necessary products in Propositions 3 and 4 and after that it willformulate the SRI product of two tensor SHs.

\section{Proposition 3}

$$
\begin{gathered}
\left(\frac{\partial^{2} P_{n^{\prime} m^{\prime}}}{\partial \theta^{2}}-\cot \theta \frac{\partial P_{n^{\prime} m^{\prime}}}{\partial \theta}+m^{\prime 2} \frac{P_{n^{\prime} m^{\prime}}}{\sin ^{2} \theta}\right)\left(\frac{\partial^{2} P_{n^{\prime \prime} m^{\prime \prime}}}{\partial \theta^{2}}-\cot \theta \frac{\partial P_{n^{\prime \prime} m^{\prime \prime}}}{\partial \theta}+m^{\prime \prime 2} \frac{P_{n^{\prime \prime} m^{\prime \prime}}}{\sin ^{2} \theta}\right)= \\
=\sum_{n=\left|n^{\prime}-n^{\prime \prime}\right|}^{n^{\prime}+n^{\prime \prime}} \Omega_{n^{\prime} m^{\prime} n^{\prime \prime} m^{\prime \prime}}^{n m} P_{n m},
\end{gathered}
$$

where

$$
\left|n^{\prime}-n^{\prime \prime}\right|<n<n^{\prime}+n^{\prime \prime}
$$




$$
\Omega_{n^{\prime} m^{\prime} n^{\prime \prime} m^{\prime \prime}}^{n m}=\left\{\begin{array}{cc}
c_{n^{\prime} m^{\prime}}^{1} c_{n^{\prime \prime} m^{\prime \prime}}^{1} Q_{n^{\prime}, m^{\prime}-2, n^{\prime \prime}, m^{\prime \prime}-2}^{n m} & m=m^{\prime}+m^{\prime \prime}-4 \\
c_{n^{\prime} m^{\prime} m^{\prime}}^{1} c_{n^{\prime \prime} m^{\prime \prime}}^{2} Q_{n^{\prime}, m^{\prime}-2, n^{\prime \prime}, m^{\prime \prime}}^{n m}+c_{n^{\prime} m^{\prime}}^{2} c_{n^{\prime \prime} m^{\prime \prime}}^{1} Q_{n^{\prime} m^{\prime} n^{\prime \prime}, m^{\prime \prime}-2}^{n m} & m=m^{\prime}+m^{\prime \prime}-2 \\
c_{n^{\prime} m^{\prime} m^{2}}^{2} c_{n^{\prime \prime} m^{\prime \prime}}^{2} Q_{n^{\prime} m^{\prime} n^{\prime \prime} m^{\prime \prime}}^{n m}+c_{n^{\prime} m^{\prime}}^{1} c_{n^{\prime \prime} m^{\prime \prime}}^{3} Q_{n^{\prime}, m^{\prime}-2, n^{\prime \prime}, m^{\prime \prime}+2}^{n m}+ & \\
+c_{n^{\prime} m^{\prime}}^{3} c_{n^{\prime \prime} m^{\prime \prime}}^{1} Q_{n^{\prime}, m^{\prime}+2, n^{\prime \prime}, m^{\prime \prime}-2}^{n m} & m=m^{\prime}+m^{\prime \prime} \\
c_{n^{\prime} m^{\prime}}^{2} c_{n^{\prime \prime} m^{\prime \prime}}^{3} Q_{n^{\prime} m^{\prime} n^{\prime \prime}, m^{\prime \prime}+2}^{n m}+c_{n^{\prime} m^{\prime}}^{3} c_{n^{\prime \prime} m^{\prime \prime}}^{2} Q_{n^{\prime}, m^{\prime}+2, n^{\prime \prime} m^{\prime \prime}}^{n m} & m=m^{\prime}+m^{\prime \prime}+2 \\
c_{n^{\prime} m^{\prime} m^{\prime}}^{3} c_{n^{\prime \prime} m^{\prime \prime}}^{3} Q_{n^{\prime}, m^{\prime}+2, n^{\prime \prime}, m^{\prime \prime}+2}^{n} & m=m^{\prime}+m^{\prime \prime}+4
\end{array} .\right.
$$

Proof. According to Eq. (4c) we can write

$$
\begin{aligned}
& \left(\frac{\partial^{2} P_{n^{\prime} m^{\prime}}}{\partial \theta^{2}}-\cot \theta \frac{\partial P_{n^{\prime} m^{\prime}}}{\partial \theta}+m^{\prime 2} \frac{P_{n^{\prime} m^{\prime}}}{\sin ^{2} \theta}\right)\left(\frac{\partial^{2} P_{n^{\prime \prime} m^{\prime \prime}}}{\partial \theta^{2}}-\cot \theta \frac{\partial P_{n^{\prime \prime} m^{\prime \prime}}}{\partial \theta}+m^{\prime \prime 2} \frac{P_{n^{\prime \prime} m^{\prime \prime}}}{\sin ^{2} \theta}\right)= \\
& =\left\{c_{n^{\prime} m^{\prime}}^{1} P_{n^{\prime}, m^{\prime}-2}+c_{n^{\prime} m^{\prime}}^{2} P_{n^{\prime} m^{\prime}}+c_{n^{\prime} m^{\prime}}^{3} P_{n^{\prime}, m^{\prime}+2}\right\}\left\{c_{n^{\prime \prime} m^{\prime \prime}}^{1} P_{n^{\prime \prime}, m^{\prime \prime}-2}+c_{n^{\prime \prime} m^{\prime \prime}}^{2} P_{n^{\prime \prime} m^{\prime \prime}}+c_{n^{\prime \prime} m^{\prime \prime}}^{3} P_{n^{\prime \prime}, m^{\prime \prime}+2}\right\} \\
& =c_{n^{\prime} m}^{1}, c_{n^{\prime \prime} m^{\prime \prime}}^{1} P_{n^{\prime}, m^{\prime}-2} P_{n^{\prime \prime}, m^{\prime \prime}-2}+c_{n^{\prime} m^{\prime}}^{1} c_{n^{\prime \prime} m^{\prime \prime}}^{2} P_{n^{\prime}, m^{\prime}-2} P_{n^{\prime \prime} m^{\prime \prime}}+c_{n^{\prime} m^{\prime}}^{1} c_{n^{\prime \prime} m^{\prime \prime}}^{3} P_{n^{\prime}, m^{\prime}-2} P_{n^{\prime \prime}, m^{\prime \prime}+2}+ \\
& +c_{n^{\prime} m^{\prime}}^{2} c_{n^{\prime \prime} m^{\prime \prime}}^{1} P_{n^{\prime} m^{\prime}} P_{n^{\prime \prime}, m^{\prime \prime}-2}+c_{n^{\prime} m^{\prime}}^{2} c_{n^{\prime \prime} m^{\prime \prime}}^{2} P_{n^{\prime} m^{\prime}} P_{n^{\prime \prime} m^{\prime \prime}}+c_{n^{\prime} m}^{2} c_{n^{\prime \prime} m^{\prime \prime}}^{3} P_{n^{\prime} m^{\prime}} P_{n^{\prime \prime}, m^{\prime \prime}+2}+ \\
& +c_{n^{\prime} m^{\prime}}^{3} c_{n^{\prime \prime} m^{\prime \prime}}^{1} P_{n^{\prime}, m^{\prime}+2} P_{n^{\prime \prime}, m^{\prime \prime}-2}+c_{n^{\prime} m^{\prime}}^{3}, c_{n^{\prime \prime} m^{\prime \prime}}^{2} P_{n^{\prime}, m^{\prime}+2} P_{n^{\prime \prime} m^{\prime \prime}}+c_{n^{\prime} m^{\prime}}^{3} c_{n^{\prime \prime} m^{\prime \prime}}^{3} P_{n^{\prime}, m^{\prime}+2} P_{n^{\prime \prime}, m^{\prime \prime}+2} .
\end{aligned}
$$

According to Eq. (10) and after further simplifications we obtain:

$$
\begin{aligned}
& \left(\frac{\partial^{2} P_{n^{\prime} m^{\prime}}}{\partial \theta^{2}}-\cot \theta \frac{\partial P_{n^{\prime} m^{\prime}}}{\partial \theta}+m^{\prime 2} \frac{P_{n^{\prime} m^{\prime}}}{\sin ^{2} \theta}\right)\left(\frac{\partial^{2} P_{n^{\prime \prime} m^{\prime \prime}}}{\partial \theta^{2}}-\cot \theta \frac{\partial P_{n^{\prime \prime} m^{\prime \prime}}}{\partial \theta}+m^{\prime \prime 2} \frac{P_{n^{\prime \prime} m^{\prime \prime}}}{\sin ^{2} \theta}\right)= \\
& =\sum_{n=n^{\prime}-n^{\prime \prime} \mid}^{n^{\prime}+n^{\prime \prime}}\left(c_{n^{\prime} m^{\prime}}^{1} c_{n^{\prime \prime} m^{\prime \prime}}^{1} Q_{n^{\prime}, m^{\prime}-2, n^{\prime \prime} m^{\prime \prime}-2}^{n m}+c_{n^{\prime} m^{\prime}}^{1} c_{n^{\prime \prime} m^{\prime \prime}}^{2} Q_{n^{\prime}, m^{\prime}-2, n^{\prime \prime} m^{\prime \prime}}^{n m}+c_{n^{\prime} m^{\prime}}^{1} c_{n^{\prime \prime} m^{\prime \prime}}^{3} Q_{n^{\prime}, m^{\prime}-2, n^{\prime \prime} m^{\prime \prime}+2}^{n m}+\right. \\
& +c_{n^{\prime} m^{2}}^{2} c_{n^{\prime \prime} m^{\prime \prime}}^{1} Q_{n^{\prime} m^{\prime} n^{\prime \prime}, m^{\prime \prime}-2}^{n m}+c_{n^{\prime} m^{\prime}}^{2}, c_{n^{\prime \prime} m^{\prime \prime}}^{2} Q_{n^{\prime} m^{\prime} n^{\prime \prime} m^{\prime \prime}}^{n m}+c_{n^{\prime} m^{\prime}}^{2} c_{n^{\prime \prime} m^{\prime \prime}}^{3} Q_{n^{\prime} m^{\prime} n^{\prime \prime}, m^{\prime \prime}+2}^{n m}+ \\
& \left.+c_{n^{\prime} m^{\prime}}^{3}, c_{n^{\prime \prime} m^{\prime \prime}}^{1} Q_{n^{\prime}, m^{\prime}+2, n^{\prime \prime}, m^{\prime \prime}-2}^{n m}+c_{n^{\prime} m}^{3} c_{n^{\prime \prime} m^{\prime}}^{2} Q_{n^{\prime}, m^{\prime}+2, n^{\prime \prime} m^{\prime \prime}}^{n m}+c_{n^{\prime} m^{\prime}}^{3}, c_{n^{\prime \prime} m^{\prime \prime}}^{3} Q_{n^{\prime}, m^{\prime}+2, n^{\prime \prime}, m^{\prime \prime}+2}^{n m}\right) P_{n m} \text {.Q.E.D. }
\end{aligned}
$$

\section{Proposition 4}

$$
\frac{\partial}{\partial \theta}\left(\frac{P_{n^{\prime} m^{\prime}}}{\sin \theta}\right) \frac{\partial}{\partial \theta}\left(\frac{P_{n^{\prime \prime} m^{\prime \prime}}}{\sin \theta}\right)=\sum_{n=\left|n^{\prime}-n^{\prime \prime}-2\right|}^{n^{\prime}+n^{\prime \prime}+2}\left(\Lambda^{v}\right)_{n^{\prime} m^{\prime} n^{\prime \prime} m^{\prime \prime}}^{n m} P_{n m}, v=1,2,3 \text { or } 4
$$

where

$$
\left|n^{\prime}-n^{\prime \prime}\right|<n<n^{\prime}+n^{\prime \prime}-2
$$




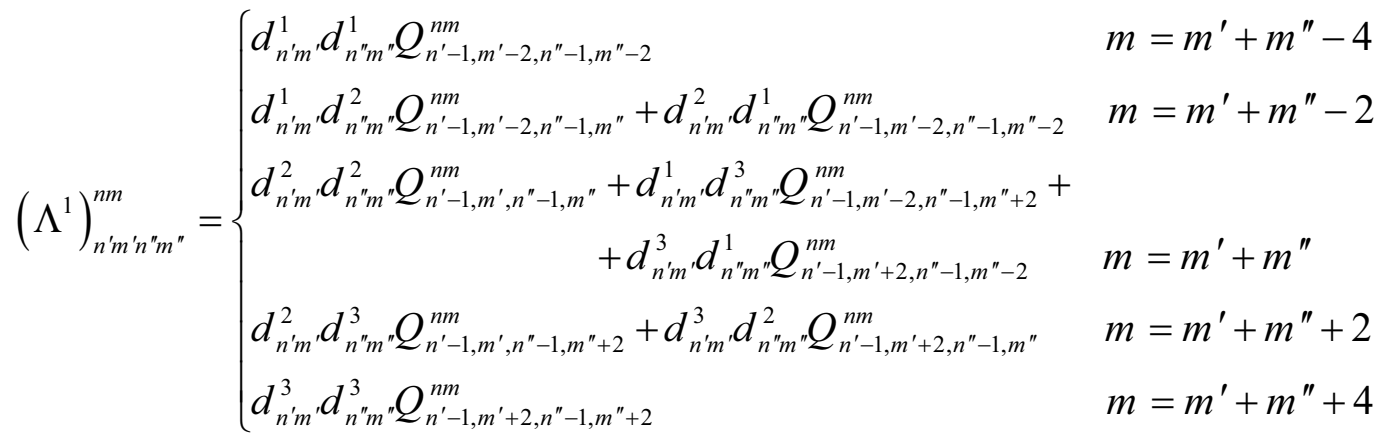

or

$$
\left|n^{\prime}-n^{\prime \prime}+2\right|<n<n^{\prime}+n^{\prime \prime}
$$

$$
\left(\Lambda^{2}\right)_{n^{\prime} m^{\prime} n^{\prime \prime} m^{\prime \prime}}^{n m}=\left\{\begin{array}{rr}
d_{n^{\prime} m^{\prime}}^{\prime 1} d_{n^{\prime \prime} m^{\prime \prime}}^{1} Q_{n^{\prime}-1, m^{\prime}-2, n^{\prime \prime}+1, m^{\prime \prime}-2}^{n m} & m=m^{\prime}+m^{\prime \prime}-4 \\
d_{n^{\prime} m^{\prime}}^{\prime 1} d_{n^{\prime \prime} m^{\prime \prime}}^{2} Q_{n^{\prime}-1, m^{\prime}-2, n^{\prime \prime}+1, m^{\prime \prime}}^{n m}+d_{n^{\prime} m^{\prime}}^{\prime 2} d_{n^{\prime \prime} m^{\prime \prime}}^{1} Q_{n^{\prime}-1, m^{\prime}-2, n^{\prime \prime}+1, m^{\prime \prime}-2}^{n m} & m=m^{\prime}+m^{\prime \prime}-2 \\
d_{n^{\prime} m^{\prime}}^{\prime 2} d_{n^{\prime \prime} m^{\prime \prime}}^{2} Q_{n^{\prime}-1, m^{\prime}, n^{\prime \prime}+1, m^{\prime \prime}}^{n m}+d_{n^{\prime} m^{\prime}}^{\prime \prime} d_{n^{\prime \prime} m^{\prime \prime}}^{3} Q_{n^{\prime}-1, m^{\prime}-2, n^{\prime \prime}+1, m^{\prime \prime}+2}^{n m}+ & \\
+d_{n^{\prime} m^{\prime}}^{\prime 3} d_{n^{\prime \prime} m^{\prime \prime}}^{1} Q_{n^{\prime}-1, m^{\prime}+2, n^{\prime \prime}+1, m^{\prime \prime}-2}^{n m} & m=m^{\prime}+m^{\prime \prime} \\
d_{n^{\prime} m^{\prime}}^{\prime 2} d_{n^{\prime \prime} m^{\prime \prime}}^{3} Q_{n^{\prime}-1, m^{\prime}, n^{\prime \prime}+1, m^{\prime \prime}+2}^{n+}+d_{n^{\prime} m^{\prime}}^{\prime 3} d_{n^{\prime \prime} m^{\prime}}^{2} Q_{n^{\prime}-1, m^{\prime}+2, n^{\prime \prime}+1, m^{\prime \prime}}^{n m} & m=m^{\prime}+m^{\prime \prime}+2 \\
d_{n^{\prime} m^{\prime}}^{\prime 3} d_{n^{\prime \prime} m^{\prime \prime}}^{3} Q_{n^{\prime}-1, m^{\prime}+2, n^{\prime \prime}+1, m^{\prime \prime}+2}^{n m} & m=m^{\prime}+m^{\prime \prime}+4
\end{array}\right.
$$

or

$$
\left|n^{\prime}-n^{\prime \prime}-2\right|<n<n^{\prime}+n^{\prime \prime}
$$

$$
=\left\{\begin{array}{cc}
d_{n^{\prime} m^{\prime}}^{1} d_{n^{\prime \prime} m^{\prime \prime}}^{\prime \prime} Q_{n^{\prime}+1, m^{\prime}-2, n^{\prime \prime}-1, m^{\prime \prime}-2}^{n m} & m=m^{\prime}+m^{\prime \prime}-4 \\
d_{n^{\prime} m^{\prime}}^{1} d_{n^{\prime \prime} m^{\prime \prime}}^{\prime 2} Q_{n^{\prime}+1, m^{\prime}-2, n^{\prime \prime}-1, m^{\prime \prime}}^{n m}+d_{n^{\prime} m^{\prime}}^{2} d_{n^{\prime \prime} m^{\prime \prime}}^{\prime \prime} Q_{n^{\prime}+1, m^{\prime}-2, n^{\prime \prime}-1, m^{\prime \prime}-2}^{n m} & m=m^{\prime}+m^{\prime \prime}-2 \\
d_{n^{\prime} m^{\prime}}^{2} d_{n^{\prime \prime} m^{\prime \prime}}^{\prime 2} Q_{n^{\prime}+1, m^{\prime}, n^{\prime \prime}-1, m^{\prime \prime}}^{n m}+d_{n^{\prime} m^{\prime}}^{1} d_{n^{\prime \prime} m^{\prime \prime}}^{\prime \prime} Q_{n^{\prime}+1, m^{\prime}-2, n^{\prime \prime}-1, m^{\prime \prime}+2}^{n m}+ & \\
+d_{n^{\prime} m^{\prime}}^{3} d_{n^{\prime \prime} m^{\prime \prime}}^{\prime 1} Q_{n^{\prime}+1, m^{\prime}+2, n^{\prime \prime}-1, m^{\prime \prime}-2}^{n m} & m=m^{\prime}+m^{\prime \prime} \\
d_{n^{\prime} m^{\prime}}^{2}, d_{n^{\prime \prime} m^{\prime \prime}}^{\prime 3} Q_{n^{\prime}+1, m^{\prime}, n^{\prime \prime}-1, m^{\prime \prime}+2}^{n m}+d_{n^{\prime} m^{\prime}}^{3} d_{n^{\prime \prime} m^{\prime \prime}}^{\prime 2} Q_{n^{\prime}+1, m^{\prime}+2, n^{\prime \prime}-1, m^{\prime \prime}}^{n m} & m=m^{\prime}+m^{\prime \prime}+2 \\
d_{n^{\prime} m^{\prime}}^{3}, d_{n^{\prime \prime} m^{\prime \prime}}^{\prime 3} Q_{n^{\prime}+1, m^{\prime}+2, n^{\prime \prime}-1, m^{\prime \prime}+2}^{n m} & m=m^{\prime}+m^{\prime \prime}+4
\end{array}\right.
$$

or

$$
\begin{aligned}
& \left|n^{\prime}-n^{\prime \prime}\right|<n<n^{\prime}+n^{\prime \prime}+2 \\
& \left(\Lambda^{4}\right)_{n^{\prime} m^{\prime} n^{\prime \prime} m^{\prime \prime}}^{n m}=\left\{\begin{array}{rr}
d_{n^{\prime} m^{\prime}}^{\prime 1} d_{n^{\prime \prime} m^{\prime \prime}}^{\prime \prime} Q_{n^{\prime}+1, m^{\prime}-2, n^{\prime \prime}+1, m^{\prime \prime}-2}^{n m} & m=m^{\prime}+m^{\prime \prime}-4 \\
d_{n^{\prime} m^{\prime}}^{\prime 1} d_{n^{\prime \prime} m^{\prime \prime}}^{\prime 2} Q_{n^{\prime}+1, m^{\prime}-2, n^{\prime \prime}+1, m^{\prime \prime}}^{n m}+d_{n^{\prime} m^{\prime}}^{\prime 2} d_{n^{\prime \prime} m^{\prime \prime}}^{\prime 1} Q_{n^{\prime}+1, m^{\prime}-2, n^{\prime \prime}+1, m^{\prime \prime}-2}^{n m} & m=m^{\prime}+m^{\prime \prime}-2 \\
d_{n^{\prime} m^{\prime}}^{\prime 2} d_{n^{\prime \prime} m^{\prime \prime}}^{\prime 2} Q_{n^{\prime}+1, m^{\prime}, n^{\prime \prime}+1, m^{\prime \prime}}^{n m}+d_{n^{\prime} m^{\prime}}^{\prime 1} d_{n^{\prime \prime} m^{\prime \prime}}^{\prime 3} Q_{n^{\prime}+1, m^{\prime}-2, n^{\prime \prime}+1, m^{\prime \prime}+2}^{n m}+ & \\
+d_{n^{\prime} m^{\prime}}^{\prime 3} d_{n^{\prime \prime} m^{\prime \prime}}^{\prime 1} Q_{n^{\prime}+1, m^{\prime}+2, n^{\prime \prime}+1, m^{\prime \prime}-2}^{n m} & m=m^{\prime}+m^{\prime \prime} \\
d_{n^{\prime} m^{\prime}}^{\prime 2} d_{n^{\prime \prime} m^{\prime \prime}}^{\prime 3} Q_{n^{\prime}+1, m^{\prime}, n^{\prime \prime}+1, m^{\prime \prime}+2}^{n n}+d_{n^{\prime} m^{\prime}}^{\prime 3} d_{n^{\prime \prime} m^{\prime \prime}}^{\prime 2} Q_{n^{\prime}+1, m^{\prime}+2, n^{\prime \prime}+1, m^{\prime \prime}}^{n m} & m=m^{\prime}+m^{\prime \prime}+2 \\
d_{n^{\prime} m^{\prime}}^{\prime 3} d_{n^{\prime \prime} m^{\prime \prime}}^{\prime 3} Q_{n^{\prime}+1, m^{\prime}+2, n^{\prime \prime}+1, m^{\prime \prime}+2}^{n n} & m=m^{\prime}+m^{\prime \prime}+4
\end{array} .\right.
\end{aligned}
$$

Proof. According to Lemmas 1 and 2 we can write: 


$$
\begin{aligned}
\frac{\partial}{\partial \theta}\left(\frac{P_{n^{\prime} m^{\prime}}}{\sin \theta}\right) \frac{\partial}{\partial \theta}\left(\frac{P_{n^{\prime \prime} m^{\prime \prime}}}{\sin \theta}\right) & =\left\{d_{n^{\prime} m^{\prime}}^{1} P_{n^{\prime}-1, m^{\prime}-2}+d_{n^{\prime} m^{\prime}}^{2} P_{n^{\prime}-1, m^{\prime}}+d_{n^{\prime} m^{\prime}}^{3} P_{n^{\prime}-1, m^{\prime}+2}\right\} \times \\
& \times\left\{d_{n^{\prime \prime} m^{\prime \prime}}^{1} P_{n^{\prime \prime}-1, m^{\prime \prime}-2}+d_{n^{\prime \prime} m^{\prime \prime}}^{2} P_{n^{\prime \prime}-1, m^{\prime \prime}}+d_{n^{\prime \prime} m^{\prime \prime}}^{3} P_{n^{\prime \prime}-1, m^{\prime \prime}+2}\right\},
\end{aligned}
$$

After expansion and simplifications we have:

$$
\begin{aligned}
& \quad \frac{\partial}{\partial \theta}\left(\frac{P_{n^{\prime} m^{\prime}}}{\sin \theta}\right) \frac{\partial}{\partial \theta}\left(\frac{P_{n^{\prime \prime} m^{\prime \prime}}}{\sin \theta}\right)= \\
& =\sum_{n=\left|n^{\prime}-n^{\prime \prime}\right|}^{n^{\prime}+n^{\prime \prime}-2}\left(d_{n^{\prime} m^{\prime}}^{1} d_{n^{\prime \prime} m^{\prime \prime}}^{1} Q_{n^{\prime}, m^{\prime}-2, n^{\prime \prime}, m^{\prime \prime}-2}^{n m}+d_{n^{\prime} m^{\prime}}^{1} d_{n^{\prime \prime} m^{\prime \prime}}^{2} Q_{n^{\prime}, m^{\prime}-2, n^{\prime \prime} m^{\prime \prime}}^{n m}+d_{n^{\prime} m^{\prime}}^{1} d_{n^{\prime \prime} m^{\prime \prime}}^{3} Q_{n^{\prime}, m^{\prime}-2, n^{\prime \prime}, m^{\prime \prime}+2}^{n m}+\right. \\
& +d_{n^{\prime} m^{\prime}}^{2} d_{n^{\prime \prime} m^{\prime \prime}}^{1} Q_{n^{\prime} m^{\prime} n^{\prime \prime}, m^{\prime \prime}-2}^{n m}+d_{n^{\prime} m^{\prime}}^{2} d_{n^{\prime \prime} m^{\prime \prime}}^{2} Q_{n^{\prime} m^{\prime} n^{\prime \prime} m^{\prime \prime}}^{n m}+d_{n^{\prime} m^{\prime}}^{2} d_{n^{\prime \prime} m^{\prime \prime}}^{3} Q_{n^{\prime} m^{\prime} n^{\prime \prime}, m^{\prime \prime}+2}^{n m}+ \\
& \left.+d_{n^{\prime} m^{\prime}}^{3} d_{n^{\prime \prime} m^{\prime \prime}}^{1} Q_{n^{\prime}, m^{\prime}+2, n^{\prime \prime}, m^{\prime \prime}-2}^{n m}+d_{n^{\prime} m^{\prime}}^{3} d_{n^{\prime \prime} m^{\prime \prime}}^{2} Q_{n^{\prime}, m^{\prime}+2, n^{\prime \prime} m^{\prime \prime}}^{n m}+d_{n^{\prime} m^{\prime}}^{3} d_{n^{\prime \prime} m^{\prime \prime}}^{3} Q_{n^{\prime}, m^{\prime}+2, n^{\prime \prime}, m^{\prime \prime}+2}^{n m}\right) P_{n m}
\end{aligned}
$$

Simplification of Eq. (16b) delivers $\left(\Lambda^{1}\right)_{n^{\prime} m^{\prime} n^{\prime \prime} m}^{n m}$. Here we proved the proposition according to Lemma 2. If we consider Lemmas 2 and 3 we can obtain further combinations which will be simplified to $\left(\Lambda^{2}\right)_{n^{\prime} m^{\prime} n^{\prime \prime} m^{\prime \prime}}^{n m}$ and $\left(\Lambda^{3}\right)_{n^{\prime} m^{\prime} n^{\prime \prime} m^{\prime \prime}}^{n m}$, respectively. If just Lemma 3 is considered we will obtain $\left(\Lambda^{4}\right)_{n^{\prime} m^{\prime} n^{\prime \prime} m^{\prime \prime}}^{n m}$.

The SRI of two tensor SHs in Eq. (8c) can be rewritten by:

$$
\iint_{\sigma_{0}} \mathbf{Z}_{n^{\prime} m^{\prime}}^{(3)}(Q):\left[\mathbf{Z}_{n^{\prime \prime} m^{\prime \prime}}^{(3)}(Q)\right]^{*} d \sigma=\iint_{\sigma_{0}}\left[G_{n^{\prime} m^{\prime}}(Q) G_{n^{\prime \prime} m^{\prime \prime}}^{*}(Q)+H_{n^{\prime} m^{\prime}}(Q) H_{n^{\prime \prime} m^{\prime \prime}}^{*}(Q)\right] d \sigma .
$$

It is straightforward to show that

$$
\begin{aligned}
\iint_{\sigma_{0}} G_{n^{\prime} m^{\prime}}(Q) G_{n^{\prime \prime} m^{\prime \prime}}^{*}(Q) d \sigma & =\frac{e^{i \Delta m \lambda_{2}}-e^{i \Delta m \lambda_{1}}}{i \Delta m} \int_{\theta_{1}}^{\theta_{2}}\left(\frac{\partial^{2} P_{n^{\prime} m^{\prime}}}{\partial \theta^{2}}-\cot \theta \frac{\partial P_{n^{\prime} m^{\prime}}}{\partial \theta}+m^{\prime 2} \frac{P_{n^{\prime} m^{\prime}}}{\sin ^{2} \theta}\right) \times \\
& \times\left(\frac{\partial^{2} P_{n^{\prime \prime} m^{\prime \prime}}}{\partial \theta^{2}}-\cot \theta \frac{\partial P_{n^{\prime \prime} m^{\prime \prime}}}{\partial \theta}+m^{\prime \prime 2} \frac{P_{n^{\prime \prime} m^{\prime \prime}}}{\sin ^{2} \theta}\right) \sin \theta d \theta
\end{aligned}
$$

and

$$
\iint_{\sigma_{0}} H_{n^{\prime} m^{\prime}}(Q) H_{n^{\prime \prime} m^{\prime \prime}}^{*}(Q) d \sigma=\frac{4 m^{\prime} m^{\prime \prime}\left(e^{i \Delta m \lambda_{2}}-e^{i \Delta m \lambda_{1}}\right)}{i \Delta m} \int_{\theta_{1}}^{\theta_{2}} \frac{\partial}{\partial \theta}\left(\frac{P_{n^{\prime} m^{\prime}}}{\sin \theta}\right) \frac{\partial}{\partial \theta}\left(\frac{P_{n^{\prime \prime} m^{\prime \prime}}}{\sin \theta}\right) \sin \theta d \theta .
$$

According to Propositions 3 and 4 and after further simplifications of Eqs. (17b) and $(17 \mathrm{c})$ and substituting the results into Eq. (17a) yields: 


$$
\begin{aligned}
\iint_{\sigma_{0}} \mathbf{Z}_{n^{\prime} m^{\prime}}^{(3)}(Q):\left[\mathbf{Z}_{n^{\prime \prime} m^{\prime}}^{(3)}(Q)\right]^{*} d \sigma & =\frac{i\left(e^{i \Delta m \lambda_{1}}-e^{i \Delta m \lambda_{2}}\right)}{\Delta m} \sum_{n=\left|n^{\prime}-n^{n}-2\right|}^{n^{\prime}+n^{n+2}+2}\left[4 m^{\prime} m^{\prime \prime}\left(\Lambda^{v}\right)_{n^{\prime} m^{\prime} n^{\prime \prime} m^{\prime \prime}}^{n m}+\Omega_{n^{\prime} m^{\prime} n^{\prime \prime} m^{\prime \prime}}^{n m}\right] \times \\
& \times \int_{\theta_{1}}^{\theta_{2}} P_{n m} \sin \theta d \theta .
\end{aligned}
$$

Similar explanation for consistency of the summation limits of $\left(\Lambda^{v}\right)_{n^{\prime} m^{\prime} n^{\prime \prime} m^{\prime \prime}}^{n{ }^{\prime \prime}}$ and $\Omega_{n^{\prime} m^{\prime} n^{n} m^{\prime \prime}}^{n m}$ can be made as that stated at the end of the proof of Proposition 3.

\section{SUMMARY}

The spherical Slepian function can be used to locally concentrate the solutions of the gradiometric boundary value problems and maximize the signal energy in the desired area. The numerators of the Slepian functions (8a)-(8c) involve SRI products of scalar, vector and tensor SHs, which have complicated forms. This paper provided some necessary products in similar and simple mathematical forms in Propositions 1 to 4; and used them to find a simple mathematical solution for the SRIs of scalar, vector and tensor SHs in terms of combinations of the Gaunt coefficients and integrals of ALFs.

Acknowledgments. The author would like to appreciate Professor Lars E. Sjöberg for the discussion about the theory of spherical harmonics and their products. The Swedish National Space Board (SNSB) is cordially acknowledged for the financial support, the project No. 63/07:1. Professor W. Kosek and the unknown reviewers are cordially acknowledged for their constructive comments.

\section{APPENDIX A: TENSOR SPHERICAL HARMONICS}

Any second-order symmetric tensor $\mathbf{V}(P)$ whose components are square integrable functions can be expanded in a series of the tensor SHs $\mathbf{Z}_{n m}^{(t)}(P), t=1,2,3, \ldots, 6$ as (Martinec 2003):

$$
\mathbf{V}(P)=\sum_{n=0}^{\infty} \sum_{m=-n}^{n} \sum_{t=1}^{6} v_{n m}^{(t)} \mathbf{Z}_{n m}^{(t)}(P)
$$

where $v_{n m}^{(t)}$ are the expansion coefficients which can be obtained using the application of the orthogonality of $\mathbf{Z}_{n m}^{(t)}(P)$ (Martinec 2003):

$$
\iint_{\sigma} \mathbf{Z}_{n m}^{(t)}(P):\left[\mathbf{Z}_{n^{\prime} m^{\prime}}^{\left(t^{\prime}\right)}(P)\right]^{*} d \sigma=4 \pi\left[N_{n}^{(t)}\right]^{2} \delta_{n n^{\prime}} \delta_{m m^{\prime}} \delta_{t t^{\prime}}
$$

where 


$$
\left[N_{n}^{(t)}\right]^{2}=\left\{\begin{array}{ll}
1 & t=1 \\
\frac{1}{2} n(n+1) & t=2 \\
2(n-1) n(n+1)(n+2) & t=3 \\
2 n^{2}(n+1)^{2} & t=4 \\
\frac{1}{2} n(n+1) & t=5 \\
2(n-1) n(n+1)(n+2) & t=6
\end{array},\right.
$$

is the square norm of base functions $\mathbf{Z}_{n m}^{(t)}(P)$, the colon denotes the double-dot product of the tensors, $\sigma$ is the unit sphere of integration, $d \sigma$ is the integration element and * is the complex conjugate operator.

Equation (A.1) is very similar to the well known $\mathrm{SH}$ expansion; the main difference is related to the fact that it presents similar harmonic expansion for tensor fields. However the base functions of Eq. (A.1) are not as simple as the scalar SHs. Note that in the case where $t=1$ the tensor SHs of $\mathbf{Z}_{n m}^{(1)}(P)$ is the same with scalar SHs $Y_{n m}(P)$.

\section{APPENDIX B: THE GAUNT COEFFICIENTS}

The Gaunt coefficients have the following relation with the Clebsch-Gordan coefficients (Xu 1996):

$$
Q_{n^{\prime} m^{\prime} n^{\prime \prime} m^{\prime \prime}}^{n m}=\sqrt{\frac{\left(n^{\prime}+m^{\prime}\right) !\left(n^{\prime \prime}+m^{\prime \prime}\right) !(n-m) !}{\left(n^{\prime}-m^{\prime}\right) !\left(n^{\prime \prime}-m^{\prime \prime}\right) !(n+m) !}} C_{n^{\prime} m^{\prime} n^{\prime \prime} m^{\prime \prime}}^{n m} C_{n^{\prime} 0 n^{\prime \prime} 0}^{n 0},
$$

where

$$
C_{n^{\prime} m^{\prime} n^{\prime \prime} m^{\prime \prime}}^{n m}=(-1)^{n^{\prime}-n^{\prime \prime}+m^{\prime}} \sqrt{2 m+1}\left(\begin{array}{ccc}
n^{\prime} & n^{\prime \prime} & n \\
m^{\prime} & m^{\prime \prime} & -m
\end{array}\right)
$$

are the Clebsch-Gordan coefficients related with the Wigner3j coefficients (Varshalovich et al. 1989) showed in Eq. (B.2) by parenthesis. If we require product of two fully-normalized ALFs it is enough to replaced the non-normalize functions by the fully-normalized one and considering their normalization factor. In this case the Gaunt coefficients will have the following relation with the Clebsch-Gordan coefficients:

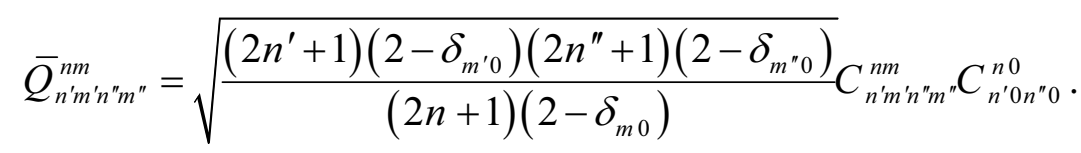




\section{REFERENCES}

Albertella A., Sansò F. and Sneeuw N. (1999) Band-limited functions on a bounded spherical domain: the Slepian problem on the sphere. Journal of Geodesy, Vol. 73, 436-447.

Albertella A., Migliaccio F. and Sansò F. (2002) GOCE: The Earth Field by Space Gradiometry. Celestial Mechanics and Dynamical Astronomy, Vol. 83, 1-15.

Balmino G., Perosanz F., Rummel R., Sneeuw N., Sünkel H. and Woodworth P. (1998) European Views on Dedicated Gravity Field Missions: GRACE and GOCE. An Earth Sciences Division Consultation Document, ESA, ESD-MAGREP-CON-001.

Balmino G., Perosanz F., Rummel R., Sneeuw N. and Sünkel H. (2001) CHAMP, GRACE and GOCE: Mission Concepts and Simulations. Bollettino di Geofisica Teoricae Applicata, Vol. 40, 3-4, 309-320.

Eshagh M. (2008) Non-singular expressions for the vector and the gradient tensor of gravitation in a geocentric spherical frame, Computers \& Geosciences, Vol. 34, $1762-1768$.

Eshagh M. (2009a) On satellite gravity gradiometry, Doctoral dissertation in Geodesy, TRITA-TEC-PHD-09004, ISSN 1653-4468Royal Institute of Technology (KTH), Stockholm, Sweden.

Eshagh M. (2009b) Complementary studies in satellite gravity gradiometry, Postdoctoral report in Geodesy, TRITA-TEC-RR 09-006, ISSN 1653-4484, ISBN 13:978-91-85539-47-5, Royal Institute of Technology (KTH), Stockholm, Sweden.

Eshagh M. (2009c) The effect of polar gaps on the solutions of gradiometric boundary value problems, Artificial Satellites, Vol. 43, No. 3, 97-108.

Eshagh M. (2010) Alternative expression for gravity gradients in local north-oriented frame and tensor spherical harmonics, Acta Geophysica, Vol. 58, 215-243.

Grünbaum F.A., Longhi Land Perlstadt M. (1982) Differential operators commuting with finite convolution integral operators: some non-abelian examples. SIAM Journal of Applied Mathematics, Vol. 42, 941-955.

Heiskanen W. and Moritz H. (1967) Physical Geodesy, W. H. Freeman and company, San Francisco and London.

Hwang C. (1991) Orthogonal Functions Over the Oceans and Applications to the Determination of Orbit Error, Geoid and Sea Surface Topography from Satellite Altimetry, PhD dissertation, JPL 958121, OSURF 720426, 229 pp, Dec, 1991.

Ilk K.H. (1983) Ein Beitrag zur Dynamik ausgedehnter KörperGravitationswechselwirkung. Deutsche Geodätische Kommission, Reihe C, Heft Nr. 288, München.

Kim M.C. and Tapley B. (2000) Formation of surface spherical harmonic normal matrices and application to high-degree geopotential modeling, Journal of Geodesy, Vol. 74, 359-375.

Koop R. (1993) Global gravity field modeling using satellite gravity gradiometry. Publ Geodesy, New series, No. 38. Netherland Geodetic Commission, Delft.

Mainville A. (1986) The altimetry-gravimetry problem using orthonormal base functions, Report No. 373, Dept. of Geod Sci, The Ohio state University, Columbus, Ohio.

Martinec Z. (2003) Green's function solution to spherical gradiometric boundaryvalue problems, Journal of Geodesy, Vol. 77, 41-49. 
Miranian L. (2004) Slepian functions on the sphere, generalized Gaussian quadrature rule. Inverse Problems, Vol. 20, 877-892.

Pail R., Plank G. and Schuh W. D. (2001) Spatially restricted data distribution on the sphere: the method of orthonormalized functions and applications, Journal of Geodesy, Vol. 75, 44-56.

Paul M.K. (1978) Recurrence relations for integrals of associated Legendre functions, BulletinGeod esique, Vol. 52, 177-190.

Rummel R. (1997) Spherical spectral properties of the Earth gravitational potential and its first and second derivatives, Geodetic boundary value problems in view of the one centimeter geoid, Lecture notes in Earth sciences Edited by Sanso F. and Rummel R., p.359-401.

Rummel R., Sanso F., Gelderen M., Koop R., Schrama E., Brovelli M., Migiliaccio F. and Sacerdote F. (1993) Spherical harmonic analysis of satellite gradiometry. Publ Geodesy, New Series, No. 39 Netherlands Geodetic Commission, Delft.

Sebilleau D. (1998) On the computation of the integrated products of three spherical harmonics, Journal of Physics A: Mathematical and General, Vol. 31, 71577168.

Slepian D. (1983) Some comments on Fourier-analysis, uncertainty and modeling, SIAM, Vol. 25, 379-393.

Simons M., Solomon S.C. and Hager B. H. (1997) Localization of gravity and topography: constraints on the tectonic and mantle dynamics of Venus, Geophysical Journal International, Vol. 131, 24-44.

Simons F.J. Dahlen F.A. and Wieczorek M.A. (2006) Spatiospectral concentration on a sphere, SIAM Review, Vol. 48, 3, 504-536.

van Gelderen M. and Rummel R. (2001) The solution of the general boundary value problem by least-squares, Journal of Geodesy, Vol. 75, 1-11.

van Gelderen M. and Rummel R. (2002) Corrections to "The solution of the general geodetic boundary value problem by least squares". Journal of Geodesy, Vol. 76, $121-122$.

Varshalovich D.A., Moskalev A.N. and Khersonskii V.K. (1989) Quantum theory of angular momentum. World Scientific Publ, Singapore.

Wieczorek M.A. and Simons F.J. (2005) Localized spectral analysis on the sphere, Geophysical Journal International, Vol. 162, 655-675.

$\mathrm{Xu}$ Y.L. (1996) Fast evaluation of the Gaunt coefficients, Mathematical Computations, Vol. 65, 1601-1612.

Zerilli F.J. (1970) Tensor harmonics in canonical form for gravitational radiation and other application. Journal of mathematical Physics, Vol. 11, 2203-2208.

Received: 2009-10-27,

Reviewed: 2010-03-24,

Accepted: 2010-03-26. 